\title{
YWHAE-NUTM2 oncoprotein regulates proliferation and cyclin D1 via RAF/MAPK and Hippo pathways
}

\author{
Wen-Bin Ou $\mathbb{1}^{1,2}$, Meijun Z. Lundberg², Shuihao Zhu', Nacef Bahri², Anastasios Kyriazoglou'², Liangliang Xu', \\ Ting Chen ${ }^{1}$, Adrian Mariño-Enriquez $\mathbb{E}^{2}$ and Jonathan A. Fletcher $\mathbb{B}^{2}$
}

\begin{abstract}
Endometrial stromal sarcoma (ESS) is the second most common subtype of uterine mesenchymal cancer, after leiomyosarcoma, and oncogenic fusion proteins are found in many ESS. Our previous studies demonstrated transforming properties and diagnostic relevance of the fusion oncoprotein YWHAE-NUTM2 in high-grade endometrial stromal sarcoma (HG-ESS) and showed that cyclin D1 is a diagnostic biomarker in these HG-ESS. However, YWHAE-NUTM2 mechanisms of oncogenesis and roles in cyclin D1 expression have not been characterized. In the current studies, we show YWHAE-NUTM2 complexes with both BRAF/RAF1 and YAP/TAZ in HG-ESS. These interactions are functionally relevant because YWHAE-NUTM2 knockdown in HG-ESS and other models inhibits RAF/MEK/MAPK phosphorylation, cyclin D1 expression, and cell proliferation. Further, cyclin D1 knockdown in HG-ESS dephosphorylates RB1 and inhibits proliferation. In keeping with these findings, we show that MEK and CDK4/6 inhibitors have anti-proliferative effects in HG-ESS, and combinations of these inhibitors have synergistic activity. These findings establish that YWHAE-NUTM2 regulates cyclin D1 expression and cell proliferation by dysregulating RAF/MEK MAPK and Hippo/YAP-TAZ signaling pathways. Recent studies demonstrate Hippo/YAP-TAZ pathway aberrations in many sarcomas, but this is among the first studies to demonstrate a well-defined oncogenic mechanism as the cause of Hippo pathway dysregulation.
\end{abstract}

\section{Introduction}

Uterine mesenchymal neoplasms afflict women across a wide age range. The biology of uterine neoplasms is heterogeneous, as exemplified by endometrial stromal sarcoma (ESS), which is the second most common subtype of malignant uterine mesenchymal tumor, after leiomyosarcoma ${ }^{1}$. Not only do ESS have varied underlying molecular oncogenic mechanisms, but these varied mechanisms are associated with differences in biologic potential, histologic appearance, and clinical behavior ${ }^{2}$. There are no standardized therapies for any of the molecularly defined subtypes of ESS, underscoring the

\footnotetext{
Correspondence: Wen-Bin Ou (ouwenbin@tsinghua.org.cn) or

Jonathan A. Fletcher (jfletcher@partners.org)

'Zhejiang Provincial Key Laboratory of Silkworm Bioreactor and Biomedicine, College of Life and Medicine, Zhejiang Sci-Tech University, 310018 Hangzhou, Zhejiang, China

2Department of Pathology, Brigham and Women's Hospital, Harvard Medical

School, 20 Shattuck Street, Thorn 528, Boston, MA 02115, USA

These authors contributed equally: Wen-Bin Ou, Meijun Z. Lundberg
}

need for biologic insights defining targetable pathways in these cancers.

Endometrial stromal neoplasms comprise several clinicopathological entities, including endometrial stromal nodules, low-grade ESS (LG-ESS), high-grade ESS (HGESS), and undifferentiated uterine sarcoma. Endometrial stromal nodules and LG-ESS often contain oncogenic fusions of JAZF1 with polycomb genes, including $S U Z 12$, PHF1, and EPC1, of which JAZF1-SUZ12 fusion is most common $^{3}$. By contrast, oncogenic polycomb gene fusions are uncommon in HG-ESS, which instead often contain YWHAE-NUTM2 fusions or BCOR intragenic mutations $^{1,4}$ : these oncogenic somatic mutations are associated with aggressive clinical behavior and poor prognosis ${ }^{5,6}$. We previously identified translocation $\mathrm{t}(10 ; 17)(\mathrm{q} 22 ; \mathrm{p} 13)$ as the mechanism of YWHAE-NUTM2 fusion in HG-ESS and we further showed the $t(10 ; 17)$ resulted in two alternative oncogenic fusions, which had been previously indistinguishable based on conventional chromosomal

\section{(c) The Author(s) 2021}

(c) (i) Open Access This article is licensed under a Creative Commons Attribution 4.0 International License, which permits use, sharing, adaptation, distribution and reproduction cc) in any medium or format, as long as you give appropriate credit to the original author(s) and the source, provide a link to the Creative Commons license, and indicate if changes were made. The images or other third party material in this article are included in the article's Creative Commons license, unless indicated otherwise in a credit line to the material. If material is not included in the article's Creative Commons license and your intended use is not permitted by statutory regulation or exceeds the permitted use, you will need to obtain permission directly from the copyright holder. To view a copy of this license, visit http://creativecommons.org/licenses/by/4.0/. 
banding studies ${ }^{1}$. These alternative oncogenic events fuse YWHAE to either of two closely related novel proteins, NUTM2A or NUTM2B, both of which are encoded by genes in $10 \mathrm{q} 22^{1}$. This is the first example of a recurrent oncogenic rearrangement involving a 14-3-3 protein in cancer and the same fusion was subsequently demonstrated in a subset of pediatric renal sarcomas ${ }^{7}$. Notably, the YWHAE-NUTM2 fusions are diagnostically specific for HG-ESS, among uterine sarcomas ${ }^{1,8}$, and are associated with cyclin D1 upregulation ${ }^{8,9}$. HG-ESS with YWHAE-NUTM2 fusion have strong nuclear cyclin D1 expression, which is found rarely - if at all - in other subtypes of ESS, and is likewise uncommon in other gynecologic sarcomas that can enter the differential diagnosis of HG-ESS, such as leiomyosarcoma ${ }^{1,9}$. We demonstrated that YWHAE-NUTM2 was not found in any of 38 LG-ESS or in 827 uterine and non-uterine mesenchymal tumors, other than HG-ESS ${ }^{1}$. Therefore, both YWHAE-NUTM2 fusion and cyclin D1 expression have proven useful as diagnostic immunomarkers for clinically-aggressive $\mathrm{ESS}^{1,9}$. However, the mechanisms by which YWHAE-NUTM2 causes cyclin D1 overexpression and HG-ESS oncogenesis have not been characterized.

The 14-3-3 protein family is encoded by seven distinct genes (YWHAB, YWHAE, YWHAG, YWHAH, YWHAQ, $Y W H A S$, and $Y W H A Z$ ) which are expressed ubiquitously in mammalian cells. All 14-3-3 proteins function as homo- or hetero-dimers and bind to motifs that contain phospho-serine or phospho-threonine residues ${ }^{10}$. These 14-3-3 interactions are primarily with signaling proteins that regulate transcription, cell cycle checkpoints, apoptosis and differentiation ${ }^{11,12}$. Both oncogenic and tumor suppressor 14-3-3 functions have been described in gynecologic cancers, but this evidence rests primarily on differences in 14-3-3 expression between the cancer cells and their nonneoplastic counterparts ${ }^{13}$. By contrast, YWHAE-NUTM2 evaluations provide the opportunity to evaluate unquestionable oncogenic 14-3-3 mechanisms in the context of a bona fide structurally-defined mutant 14-3-3 oncoprotein. YWHAE-NUTM2 retains the conserved 14-3-3 protein binding domains encoded by exons 2 and 4 of YWHAE, and thereby retains YWHAE dimerization and phosphopeptide binding properties ${ }^{1}$. These YWHAE functions are redirected from their usual predominantly cytoplasmic location to the nucleus, due to influence of a NUTM2 bipartite nuclear localization motif ${ }^{1}$.

In the studies reported herein we provide insight into how YWHAE-NUTM2 leads to cyclin D1 overexpression, thereby driving HG-ESS oncogenesis. In these studies, we evaluate whether YWHAE-NUTM2 induces HG-ESS growth and cyclin D1 expression by interacting with RAF1/BRAF and the Hippo effectors YAP/TAZ. The findings unravel oncogenic mechanisms in HG-ESS and provide rationales for targeting RAF/MEK/MAPK and Hippo-YAP/TAZ signaling pathways as therapeutic approaches in HG-ESS with YWHAE-NUTM2.

\section{Results \\ YWHAE-NUTM2 interacts with RAF1 and BRAF}

We investigated interaction of YWHAE-NUTM2 with RAF1 and BRAF in ESS1 cells expressing only the endogenous YWHAE-NUTM2 vs. expressing endogenous YWHAE-NUTM2 together with a stably-incorporated lentiviral YWHAE-NUTM2-FLAG construct. RAF1 and BRAF were immunoprecipitated from these cells, and the immunoprecipitates were then blotted and immunostained for YWHAE, FLAG, RAF1, and BRAF (Fig. 1 and SFig. 1). These studies demonstrated YWHAE-NUTM2 $140 / 110 \mathrm{kDa}$ isoform complexing with RAF1 and BRAF in ESS1 parental cells and in ESS1 expressing the YWHAENUTM2-FLAG construct (Fig. 1 and SFig. 1).

\section{YWHAE-NUTM2 regulates RAF/MEK/MAPK}

To address the hypothesis that YWHAE-NUTM2 regulates the RAF/MEK/MAPK pathway, we stably silenced YWHAE-NUTM2 in ESS1 using lentiviral shRNA constructs. Immunoblotting studies 10 days after the YWHAE-NUTM2 shRNA transductions and puromycin selection showed greater than $60 \%$ inhibition of YWHAENUTM2 expression (Fig. 2). This was accompanied by dephosphorylation of RAF1, BRAF, MEK, and MAPK, and inhibition of cyclin D1, cyclin A, and PCNA proliferation expression (Fig. 2). Further studies suggested that cyclin D1 overexpression in YWHAE-NUTM2 ESS is mediated, at least in part, by RAF1 and BRAF. Expression of these RAF kinases was inhibited ( $>70 \%$ ) by siRNAs resulted in downregulation of cyclin D1 expression (Fig. 3A). The RAF1 and BRAF siRNA-mediated knockdowns resulted,






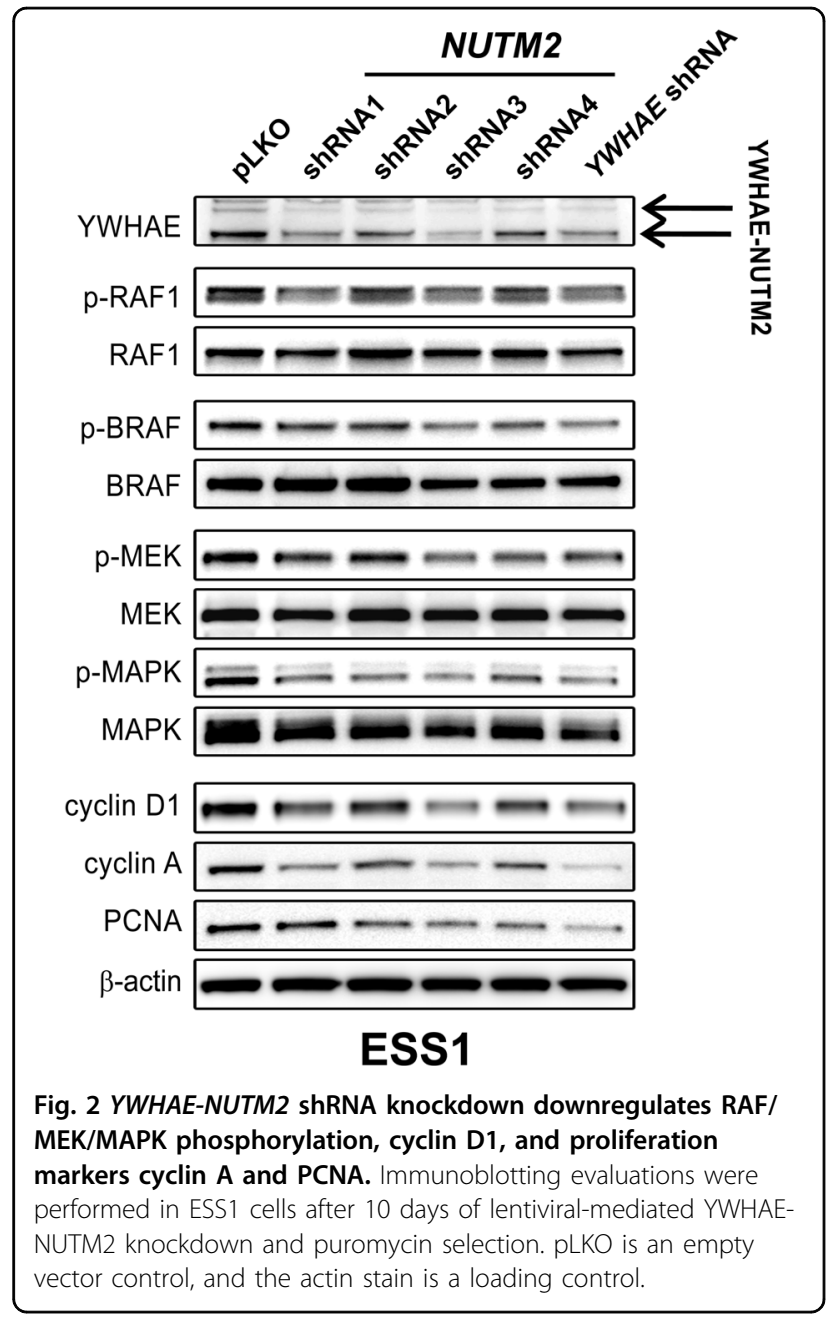

respectively, in 25 and $60 \%$ inhibition of ESS1 viability at 6 days compared with scramble siRNA controls (Promega CellTiter-Glo assay; Madison, WI, USA; Fig. 3B).

\section{YWHAE-NUTM2 regulates the Hippo pathway}

Based on precedent for YWHAE interactions with YAP and TAZ ${ }^{14-17}$, we asked whether YWHAE-NUTM2 interacts with YAP and TAZ in ESS1 cells expressing endogenous YWHAE-NUTM2, and in HEK293T and 3T3 cells $48 \mathrm{~h}$ post-transfection with YWHAE-NUTM2-FLAG or YWHAE-HA constructs. Immunoblotting of YAP and TAZ immunoprecipitations demonstrated YAP/TAZ interactions with YWHAE-NUTM2 (140 kDa band) in ESS1 cells and in the HEK293T and 3T3 cells (Fig. 4A-C and SFig. 2). Notably, YAP/TAZ interactions were not demonstrable with a comparator YWHAE-HA construct in HEK293T cells, suggesting that YAP/TAZ interactions with YWHAE-NUTM2 are stronger than those reported previously for YWHAE (Fig. 4B and SFig. 2).

\section{YWHAE-NUTM2 regulates cell viability via the Hippo pathway}

Among uterine sarcomas, cyclin D1 is strongly and uniquely overexpressed in YWHAE-NUTM2 HG-ESS 9 . Cyclin D1 is a key effector of the Hippo pathway. To evaluate whether YWHAE-NUTM2 regulates HG-ESS viability via Hippo/cyclin D1, we silenced $Y W H A E$ NUTM2, YAP, and TAZ in ESS1 with siRNAs, and then determined expression of the Hippo effectors - CTGF, CYR61, and cyclin D1 - by immunoblotting (Fig. 5A, B). siRNA transfections accomplishing $>80 \%$ downregulation of YWHAE-NUTM2, YAP, or TAZ expression
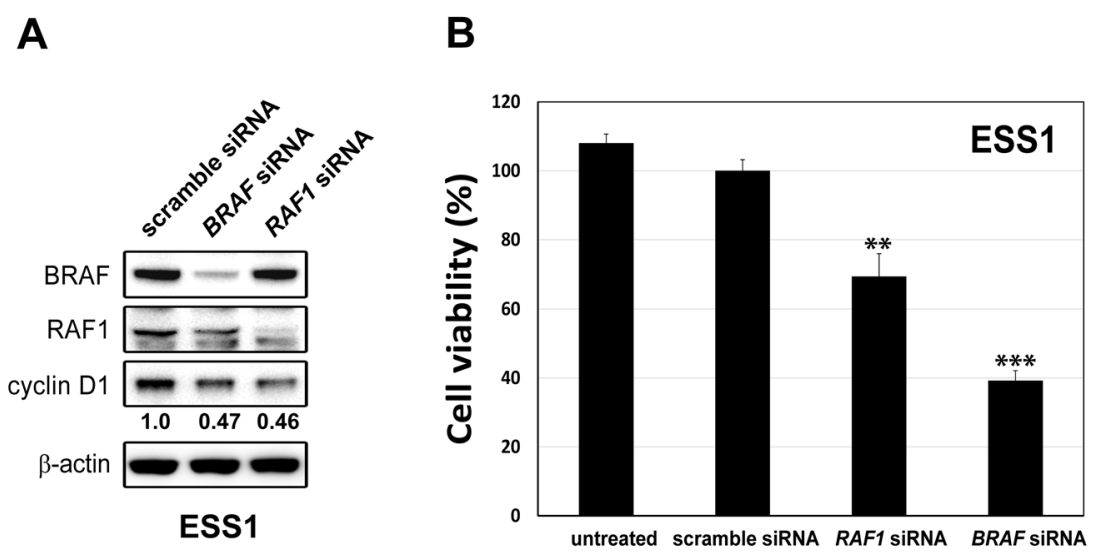

Fig. 3 BRAF and RAF1 siRNA knockdowns downregulate cyclin D1 and viability in ESS1 cells. A Immunoblotting evaluations were performed in ESS1 cells 4 days after RAF1 or BRAF siRNA transfections (each at $150 \mathrm{nM}$ ). The actin stain is a loading control. B Cell viability was determined in ESS1 cells 6 days after RAF1 or BRAF siRNA transfections (each at $150 \mathrm{nM}$ ) using a Cell-titer Glo ${ }^{\circledR}$ luminescence assay. Data were normalized to the scramble siRNA control and represent the mean values ( \pm s.d.) from quadruplicate cultures, averaged from two independent experiments. Statistically significant differences between scramble siRNA control and RAF1 siRNA or BRAF siRNA treatments are indicated as ${ }^{* *} p<0.01,{ }^{* * *} p<0.001$. 


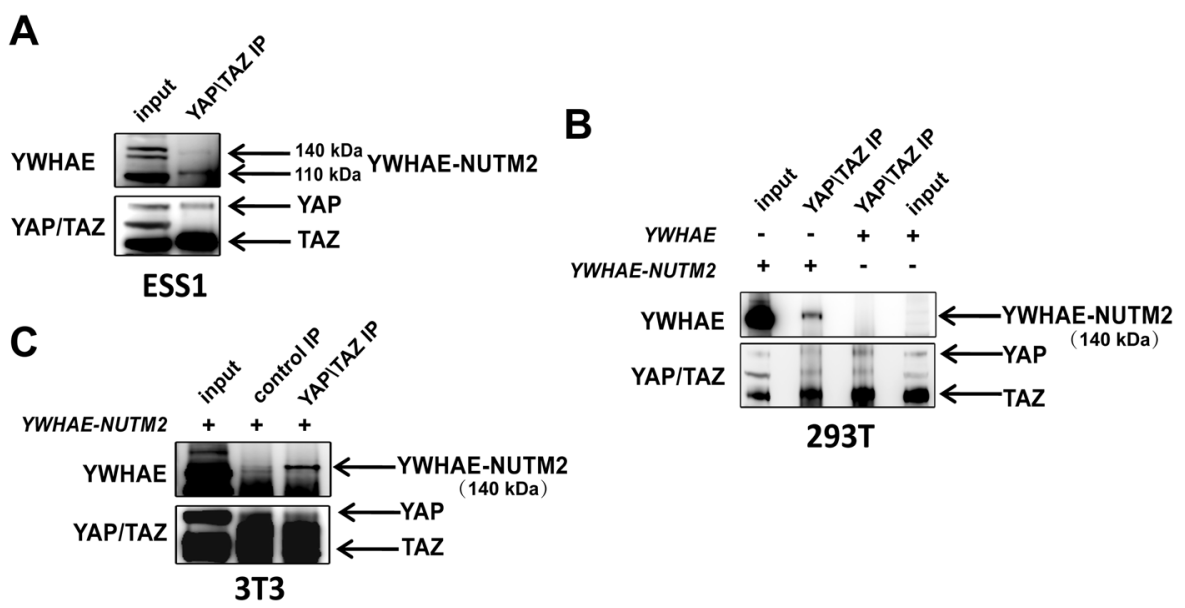

Fig. 4 YWHAE-NUTM2 interactions with Hippo pathway effectors YAP and TAZ. A YAP/TAZ immunoprecipitation in ESS1 cells immunoblotted for YWHAE. B YAP/TAZ immunoprecipitations in 293T cells, 2 days after transfections with YWHAE-NUTM2 vs. YWHAE constructs, immunoblotted for YWHAE. C YAP/TAZ immunoprecipitations in $3 T 3$ cells, 2 days after transfection with YWHAE-NUTM2. Normal rabbit serum IgG immunoprecipitation (middle lane) is a negative control.

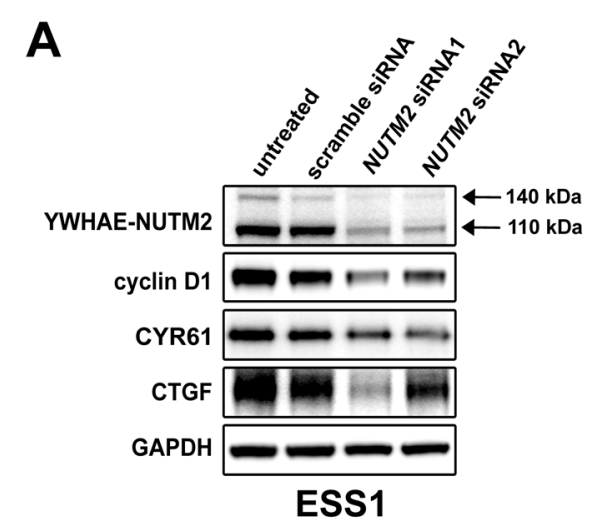

C

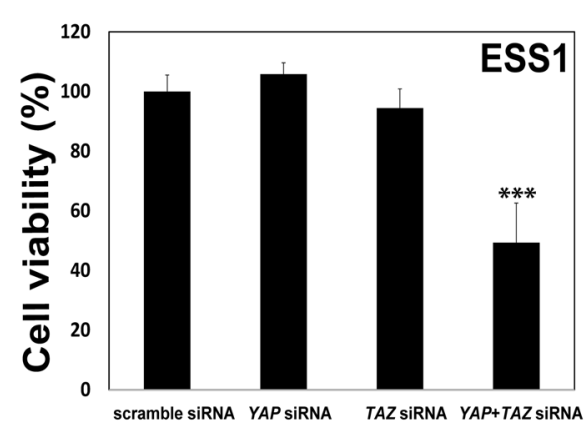

B

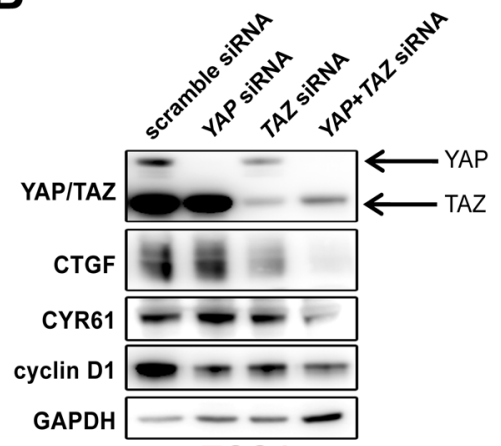

ESS1

D
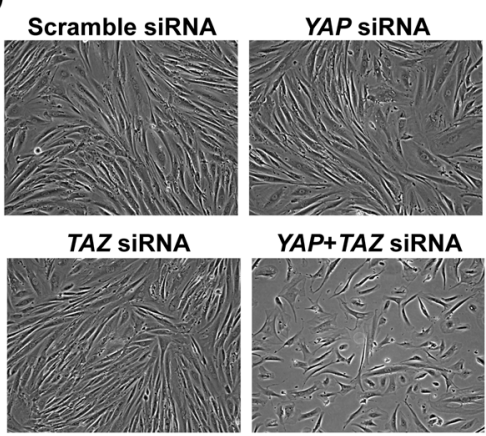

ESS1

Fig. 5 YWHAE-NUTM2 and Hippo pathway functional relationships. A Immunoblotting for Hippo effectors was performed in ESS1 cells 5 days after NUTM2 siRNA transfections (100 nM). The GAPDH stain is a loading control. B Immunoblotting was performed in ESS1 cells 5 days after YAP, TAZ, or YAP + TAZ siRNA transfections (each at $100 \mathrm{nM}$ ). The GAPDH stain is a loading control. C Cell viability was determined in ESS1 cells 6 days after YAP, $T A Z$, or YAP + TAZ siRNA transfections (each at $100 \mathrm{nM}$ ) using a Cell-titer Glo ${ }^{\circledast}$ luminescence assay. Data were normalized to scramble siRNA control and represent the mean values $( \pm$ s.d.) from quadruplicate cultures and were averaged from two independent experiments. Statistically significant differences between scramble siRNA control and YAP, TAZ, or YAP + TAZ siRNA treatments are indicated as ${ }^{* *} p<0.001$. D ESS1 growth response 5 days after YAP, TAZ, or YAP + TAZ siRNA transfections. 

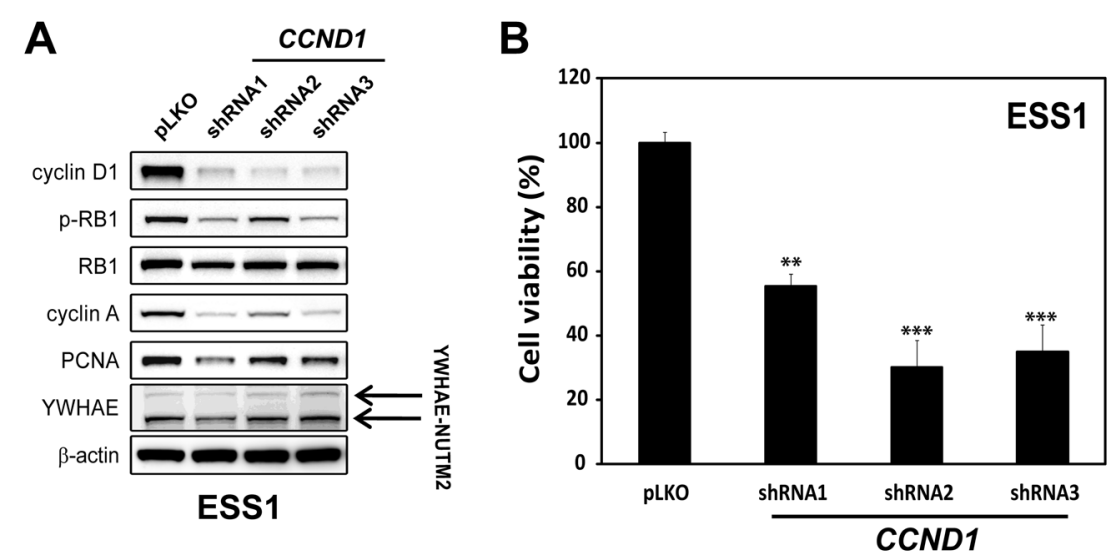

Fig. 6 Cyclin D1 regulation of HG-ESS proliferation. A Immunoblotting for RB1 dephosphorylation (RB1 activation) and proliferation markers cyclin A and PCNA were performed in ESS1 cells 4 days after CCND1 shRNA lentiviral knockdown. YAP, TAZ, or YAP + TAZ siRNA transfections (each at $100 \mathrm{nM}$ ). pLKO is an empty vector control, and the actin stain is a loading control. B Cell viability was determined in ESS1 cells 6 days after CCND1 shRNA lentiviral knockdowns with three independent shRNA sequences. The data were normalized to empty lentivirus (pLKO) control infections and represent the mean values $( \pm$ s.d.) from quadruplicate cultures, and were averaged from two independent experiments. Statistically significant differences between empty vector control and CCND1 shRNA are indicated as ${ }^{* *} p<0.01,{ }^{* *} p<0.001$.

suppressed expression of CTGF, CYR61, and cyclin D1 (Fig. 5A, B). Concomitant siRNA inhibition of both YAP and TAZ inhibited ESS1 cell viability more than inhibition of either gene individually (Fig. 5C and SFig. 3). Similarly, ESS1 six-well monolayer growth was inhibited markedly after concomitant YAP-TAZ siRNA knockdown (Fig. 5D). These studies were corroborated by three independent siRNA transfections.

\section{Cyclin D1 regulates proliferation in ESS}

Because cyclin D1 has varied biological roles, we determined whether CCND1 manipulations impacted cell growth and biomarkers of ESS cell proliferation. CCND1 gene expression was silenced by lentiviral shRNA transduction in ESS1 cells, resulting in $>90 \%$ inhibition of cyclin D1 expression, assessed at $96 \mathrm{~h}$ after transduction (Fig. 6A). This inhibition of cyclin D1 expression resulted in inhibition of RB1 phosphorylation, inhibition of proliferation markers cyclin A and PCNA (Fig. 6A), and inhibition of cell viability (Fig. 6B) and wound healing (SFigure 4). However, inhibition of cyclin D1 expression had minimal or no impact on YWAHE-NUTM2 fusion oncoprotein expression. These studies were corroborated by three independent shRNA transductions.

\section{Synergistic anti-proliferative effects via dual targeting of MEK and CDK4/6}

Synergistic antagonism of cell proliferation was demonstrated after combined inhibition of MEK and CDK4/6-cyclin D1 in ESS1 cells (Fig. 7). MEK inhibition by PD325901 (10 $\mathrm{nM})$ and CDK4/6 inhibition by palbociclib $(50 \mathrm{nM})$ resulted, respectively, in 55 and $65 \%$ reductions in ESS viability, compared to the DMSO

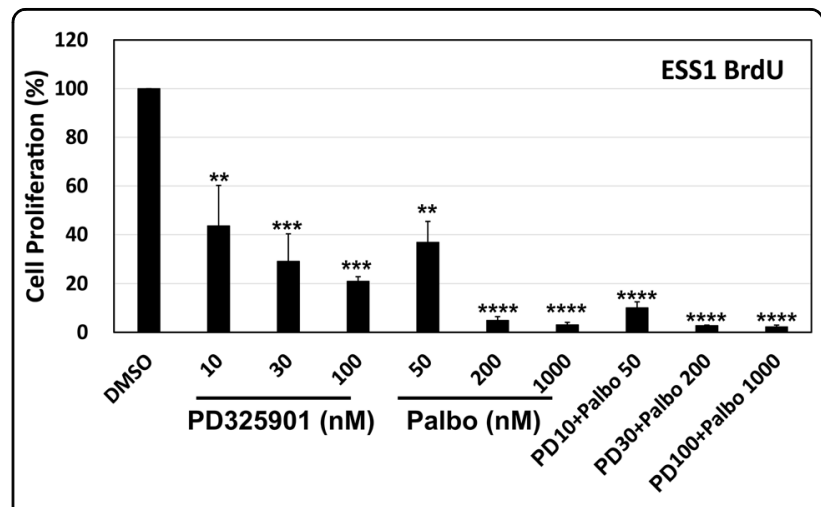

Fig. 7 MEK-inhibitor and CDK4/6-inhibitor individual and combined effects on HG-ESS proliferation. ESS1 cells were treated with PD325901, palbociclib, or combined PD325901 and palbociclib at indicated concentrations for 3 days, and labeled with anti-BrdU-POD for the last $24 \mathrm{~h}$. The data were normalized to a DMSO control and represent the mean values $( \pm$ s.d.) from quadruplicate cultures, and were averaged from two independent experiments. Statistically significant differences between DMSO control and inhibitor treatments are indicated as ${ }^{* *} p<0.01,{ }^{* *} p<0.001,{ }^{* * *} p<0.0001$.

control. Dose-response anti-proliferative effects were also demonstrated for both PD325901 and palbociclib. Combination treatment with PD325901 (10 nM) and palbociclib $(50 \mathrm{nM})$ resulted in $90 \%$ reduction in ESS1 viability (Fig. 7), ${ }^{* * *} p<0.01$ for $10 \mathrm{nM}$ PD325901 and $50 \mathrm{nM}$ palbociclib treatment alone, and ${ }^{* * * *} p<0.0001$ for combination treatment.

\section{Discussion}

These novel studies demonstrate that cyclin D1 overexpression in HG-ESS depends on YWHAE-NUTM2 
activation of RAF/MAPK and Hippo pathways. The rationales for studying RAF/MAPK pathway roles were: (1) MAPK is a known positive regulator of cyclin D1 expression $^{18}$; (2) BRAF and RAF1 heterodimerization is generally required for MEK/MAPK phosphorylation and cell proliferation ${ }^{19,20}$; (3) BRAF and RAF1 complex with YWHAE and other $14-3-3$ proteins ${ }^{19,21,22}$;, and (4) precedent for RAF1 translocation from cytoplasm to the nucleus $^{23-25}$ raised the possibility that RAF1 might interact with the predominantly nuclear YWHAENUTM2 oncoproteins. The rationales for studying Hippo pathway roles were: (1) upregulation of cyclin D1, along with CTGF and CYR61, is a well-known Hippo function, resulting from transcriptional upregulation by the Hippo effectors YAP and TAZ; (2) YAP and TAZ interact with YWHAE and other $14-3-3$ proteins $^{14-17}$; and (3) our recent studies show that Hippo YAP/TAZ mechanisms induce dramatic cyclin D1 overexpression and cell proliferation in another subtype of mesenchymal neoplasia, gastrointestinal stromal tumor ${ }^{26}$.

The studies reported herein indeed demonstrate YWHAE-NUTM2 interaction with BRAF and RAF1, and with YAP and TAZ. These interactions were demonstrated in a variety of models, including ESS1, which is the only known cell line derived from a YWHAE-NUTM2 HG-ESS (Fig. 1, SFigs. 1 and 2, and Fig. 4). Furthermore, YWHAENUTM2 knockdown in ESS1 inhibited phosphorylation of BRAF, RAF1, MEK, and MAPK, and inhibited expression of cyclin D1 and the proliferation markers cyclin A and PCNA (Fig. 2). Evidence for RAF-MEK pathway roles in HG-ESS oncogenesis included ESS1 dependence on BRAF and RAF1 for viability and cyclin D1 expression (Fig. 3A, B), and on MEK/MAPK for proliferation (Fig. 7).

The above-mentioned findings are consistent with YWHAE-NUTM2 oncogenic roles in RAF/MEK/MAPK pathway dysregulation which then induce ESS proliferation and cyclin D1 overexpression. However, our studies show that HG-ESS cyclin D1 overexpression and cell proliferation do not depend only on RAF1 and BRAF dysregulation, but also result from Hippo dysregulation. The evidence for Hippo oncogenic dysregulation as a mechanism for cyclin D1 upregulation includes inhibition of cyclin D1 and the Hippo signaling effectors CYR61 and CTGF (Fig. 5A) after YWHAE-NUTM2 silencing, together with the above-mentioned YWHAE-NUTM2 interaction with YAP/TAZ. In keeping with this evidence, YAP and TAZ knockdowns reduced ESS1 viability and inhibited expression of cyclin D1, CYR61, and CTGF (Fig. 5B-D and SFig. 3). Interestingly, YAP and TAZ are transcription factors whose nuclear localization results from novel nuclear localization signals but also from Hippo inactivation, which causes YAP/TAZ-dephosphorylation and release from cytoplasmic retention factors, particularly 14-3-3 proteins, including YWHAE ${ }^{16}$.
Cyclin D1 has varied tumorigenic roles in human cancers which include CDK4/6 complexing to regulate the cell cycle $^{27}$ and RAD51-coregulation of DNA damage repair $^{28}$. These putative oncogenic roles generally result from cyclin D1 overexpression, and the mechanisms of overexpression are largely unknown, although genomic CCND1 amplification or translocation accounts for overexpression in subsets of human cancer, including breast cancers and lympoma ${ }^{29,30}$. In the studies herein CCND1 knockdown in ESS1 substantially inhibited cell proliferation, inhibited the cyclin A and PCNA proliferation markers, and inhibited RB1 hyperphosphorylation - indicating restoration of RB1 tumor suppressor function (Fig. 6A, B). Likewise, CDK4/6 inhibition by palbociclib treatment had substantial antiproliferative effects in ESS1 (Fig. 7). Notably, additive ESS1 anti-proliferative effects resulted from combination treatment with the MEK and CDK4/6 inhibitors (Fig. 7), providing further evidence that both Hippo/cyclin D1 and RAF-MEK pathways are required for YWHAE-NUTM2 oncogenesis.

In sum, these studies provide the first insights into YWHAE-NUTM2 oncogenic mechanisms and intriguingly show that YWHAE-NUTM2 dysregulates both RAF1/BRAF and the Hippo pathway to cause overexpression of cyclin D1 (Fig. 8), which is a key diagnostic biomarker in HG-ESS. These advances also identify the RAF/MEK/MAPK and Hippo pathways, and CDK4/6, as rational targets for evaluation of therapeutic strategies in

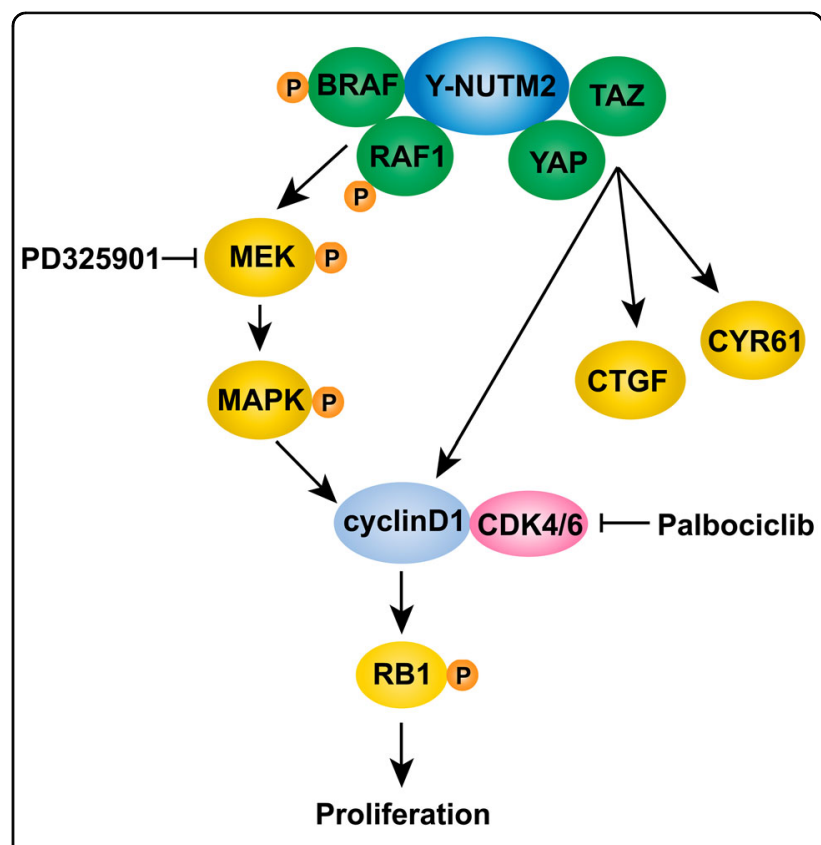

Fig. 8 YWHAE-NUTM2 interacts with BRAF/RAF1 and YAP/TAZ in high-grade ESS. YWHAE-NUTM2 oncogenic activation of these pathways regulates cyclin D1 expression and thereby cell proliferation. 
HG-ESS with YWHAE-NUTM2 fusions. Although there are no approved targeted therapies for HG-ESS, a recent study demonstrated substantial clinical response to the kinase-inhibitor pazopanib in an ESS patient with YWHAE-NUTM2 rearrangement ${ }^{31}$. The advances reported herein identify new rationales for targeted therapies in these clinically challenging cancers.

\section{Materials and methods}

\section{Antibodies and reagents}

Polyclonal antibody to YWHAE (HPA008445) was from Sigma-Aldrich (St, Louis, MO). Polyclonal antibodies to p-RB1 (S807/811, \#9308), p-RAF1 (S259, \#9421), p-BRAF (S445, \#2696), p-MEK (S217/221, \#9121), p-MAPK (T202/Y204, \#9101), MEK (\#9122), MAPK (\#9102), and YAP/TAZ (\#8418) were from Cell Signaling Technology (Beverly, MA), whereas those for RAF1 (sc-7267), CTGF (sc-14939) and CYR61 (sc-13100) were from Santa Cruz Biotechnology (Santa Cruz, CA). Monoclonal mouse antibodies for BRAF (sc-5284), cyclin D1 (sc-20044), and PCNA (sc-56) were from Santa Cruz Biotechnology, for RB1 (\#9309) was from Cell Signaling Technology, for cyclin A (NCL-CYCLIN A) was from Novocastra/Leica (Newcastle upon Tyne, UK), and for FLAG (F-1804), GAPDH (G8795), and actin (A4700) were from SigmaAldrich. Platinum PCR SuperMix was from Invitrogen Life Technologies (Carlsbad, CA). PD325901 and palbociclib were from LC Laboratories (Woburn, MA).

\section{Cell culture}

The ESS1 cell line was established from a HG-ESS with YWHAE-NUTM $2^{1}$. Cells were screened for mycoplasma using a Mycoplasma Stain Assay Kit (Sigma-Aldrich), and authenticated by SNP array analysis prior to these studies and by RT-PCR and FISH, before and after the studies, to validate presence of the YWHAE-NUTM2 rearrangement. The ESS1 cell line was maintained in IMDM medium with $15 \%$ fetal bovine serum (FBS) containing penicillin/ streptomycin and L-glutamine. The studies were conducted in accordance with recognized ethical guidelines (U.S. Common Rule) and were approved by Brigham and Women's Hospital and Zhejiang Sci-Tech University Institutional Review Boards.

\section{Fusion construct and cloning}

A YWHAE-NUTM2-FLAG fusion cDNA containing BamHI (YWHAE sequence) and EcoRI (FLAG sequence) restriction sites was synthesized (GenScript) per the YWHAE-NUTM2 fusion transcript sequence in ESS1 and cloned in a pUC57 vector. The fusion gene sequence was validated by sequencing. It was further subcloned in pCDNA3(+) by EcoRI and BamHI (GenScript). YWHAE-NUTM2-FLAG was then subcloned into lentiviral vector $\mathrm{pCDH}-\mathrm{CMV}-\mathrm{MCS}-\mathrm{EF} 1-\mathrm{Puro}$ (System
Biosciences) by Nhe1 \& Not1 (New England Biolabs). Construct integrity was verified by Sanger sequencing.

\section{Transfection}

The fusion construct was expressed in 293T or 3T3 cells by Lipofectamine-based transfection according to the manufacturer's instructions (Invitrogen Life Technologies). Briefly, scrambled control, NUTM2 siRNAs (s198355 and s195919; Invitrogen Life Technologies), BRAF siRNA (5'-AAGUGGCAUGGUGAUGUGGCA-3', Invitrogen Life Technologies), RAF1 siRNA (5'-AAUAGUUCAGCAGUUUGGCUA-3', Invitrogen Life Technologies), YAP siRNA (sc-38637), TAZ siRNA (sc-38568, Santa Cruz Biotechnology), YWHAE-NUTM2-FLAG, or $Y W H A E-H A$ constructs were incubated with PLUS in serum-free medium for $15 \mathrm{~min}$ at room temperature, then mixed in diluted Lipofectamine in equal volumes with scrambled control, siRNAs or construct-PLUS mixtures and incubated for another $15 \mathrm{~min}$ at room temperature. Finally, siRNA/construct-PLUS-Lipofectamine complexes were added to $60 \%$ confluent ESS1 cells, 293T, or 3T3 cell lines under serum-free medium conditions in 6or 96-well plates. DNA-PLUS-Lipofectamine complexes in serum-free medium were completely replaced with serum-containing regular medium after a 3 -h incubation. Cells were lysed for immunoprecipitation at $48 \mathrm{~h}$, western blot analysis at $96 \mathrm{~h}$, or cell viability assay at 6 days post-transfection. Cell culture images were obtained by using a Spot RT Slider Camera and Spot software (Version 4.6 for Windows) and a Nikon Eclipse TE2000-S inverted microscope. Experiments were performed in triplicate.

\section{Preparation of lentiviral CCND1, NUTM2, and YWHAE shRNA constructs and lentiviral infections}

Lentiviral CCND1 shRNA constructs (shRNA1, TRCN0000040038; shRNA2, TRCN0000040039; and shRNA3, TRCN0000040042) were from Sigma. NUTM2 and YWHAE shRNAs were from Broad Institute RNAi Consortium: NUTM2 shRNA1, 5'-TGCTCCTGTGGTG CCTGTTAT-3'; and NUTM2 shRNA2, 5'-GTGAGTCA GAAGGACAATTTA-3', NUTM2 shRNA3, 5'-TCTTGC TGGGCCTTAGCTTTG- $3^{\prime}$; and NUTM2 shRNA4, $5^{\prime}$-TA TGTTCCAGGAACCTGTTTA-3'. YWHAE shRNA: 5'-C CACAGGTATCTGGCAGAATT- ${ }^{\prime}$. Lentiviral preparations were produced by cotransfecting empty vector pLKO.1 puro with CCND1, NUTM2, or YWHAE shRNAs and helper virus packaging plasmids pCMV $\triangle \mathrm{R} 8.91$ and vsv-g (at a 10:10:1 ratio) into 293T cells. Transfections were carried out with Lipofectamine and PLUS reagent. Lentiviruses were harvested at $24,36,48$, and $60 \mathrm{~h}$ posttransfection and stored at $-80^{\circ} \mathrm{C}$.

ESS1 cells were seeded in six-well plates. Infections were carried out in the presence of $8 \mu \mathrm{g} / \mathrm{mL}$ polybrene. 
After transduction, ESS1 were selected with $2 \mu \mathrm{g} / \mathrm{mL}$ puromycin for 10 days, then lysed for western blot analysis.

\section{Immunoblotting}

Whole cell lysates were prepared using lysis buffer $(1 \%$ $\mathrm{NP}-40,50 \mathrm{mM}$ Tris- $\mathrm{HCl} \mathrm{pH}$ 8.0, $100 \mathrm{mM}$ sodium fluoride, $30 \mathrm{mM}$ sodium pyrophosphate, $2 \mathrm{mM}$ sodium molybdate, $5 \mathrm{mM}$ EDTA, and $2 \mathrm{mM}$ sodium orthovanadate) containing protease inhibitors $(10 \mu \mathrm{g} / \mathrm{mL}$ aprotinin, $10 \mu \mathrm{g} / \mathrm{mL}$ leupeptin, and $1 \mathrm{mM}$ phenylmethylsulfonyl fluoride). Lysates were cleared by centrifugation at $14,000 \mathrm{rpm}$ for $20 \mathrm{~min}$ at $4{ }^{\circ} \mathrm{C}$, and lysate protein concentrations were determined using a Bio-Rad protein assay (Bio-Rad Laboratories Hercules, CA, USA). Electrophoresis and western blotting were performed as described previously ${ }^{32}$. The hybridization signals were detected by chemiluminescence (Immobilon Western, Millipore Corporation, MA) and captured using an Amersham Imager 600 chemiluminescence imaging system (GE Healthcare, MA, USA).

\section{Immunoprecipitation}

One mg of protein lysate $(500 \mu \mathrm{L})$ was preadsorbed for 30 min using $20 \mu \mathrm{L}$ of sepharose-protein G or A beads at $4{ }^{\circ} \mathrm{C}$. Then $2 \mu \mathrm{g}$ of primary antibodies to RAF1, BRAF, or YAP/TAZ were rocked with the lysates for $2 \mathrm{~h}$ at $4{ }^{\circ} \mathrm{C}$, whereas normal mouse IgG or rabbit IgG was substituted as a comparator group for immunoprecipitation stringency. Then $20 \mu \mathrm{L}$ of sepharose-protein G/A beads were added and rocked overnight at $4{ }^{\circ} \mathrm{C}$, then centrifuged at $10,000 \mathrm{rpm}$ for $2 \mathrm{~min}$ at $4{ }^{\circ} \mathrm{C}$, after which the sepharose beads were washed three times with $750 \mu \mathrm{L}$ of IP buffer (lysis buffer without protease inhibitors) for $25 \mathrm{~min}$ per wash, and were then washed once with $750 \mu \mathrm{L} 10 \mathrm{mM}$ Tris-Cl buffer ( $\mathrm{pH}$ 7.6). Loading buffer $(20 \mu \mathrm{L})$ was added to the beads and boiled for $5 \mathrm{~min}$ at $95^{\circ} \mathrm{C}$.

\section{Cell viability analysis}

ESS1 cells were plated at 2500 cells/well in a 96-well flat-bottomed plate (Falcon, Lincoln NJ) and cultured in Iscove's modified Dulbecco's Medium for $24 \mathrm{~h}$ before treatment with siRNA. Cell viability was determined 6 days post-transfection with the CellTiter-Glo luminescent assay from Promega (Mannheim, Germany). Viability was quantified using a Veritas ${ }^{\mathrm{TM}}$ Microplate Luminometer from Turner Biosystems (Sunnyvale, CA). Data were normalized to scramble controls. All assays were performed in quadruplicate wells and in triplicate.

\section{In vitro wound-healing assays}

A slash was created in confluent cell cultures, using the tip of a P-100 pipette, at 4 days after CCND1 shRNA transduction. The plates were photographed at 0 and $72 \mathrm{~h}$ with Spot software (Version 4.6 for Windows) and a Nikon Eclipse TE2000-S inverted microscope. Experiments were performed in triplicate.

\section{BrdU uptake analysis}

Cells were plated in 96-well plates at 5000 cells/well in growth medium and incubated overnight. Cells were treated with PD325901 or palbociclib for 72 hours. BrdU was added to the cells for the last 24 hours. Cell labeling with BrdU, fixation and detection were performed using a BrdU Cell Proliferation Assay kit and anti-BrdUperoxidase (Roche Applied Science, Branford, CT). BrdU incorporation was presented as \% of DMSO control. All assays were performed in triplicate wells and were repeated three times.

\section{Statistical analysis}

Student's $t$ tests were performed on data from cells treated with inhibitors/shRNAs/siRNAs or DMSO/pLKO (control). Statistically significant differences between control and treatment were defined as ${ }^{*} p<0.05$, "** $p<$ $0.01,{ }^{* * * *} p<0.001$, and ${ }^{* * * * *} p<0.0001$.

\section{Acknowledgements}

This work was supported by the Sherwood Foundation, the SARC LMSARC research project, and by NIH grants 1P50CA127003 and 1P50CA168512. This research was also supported by the Fundamental Research Funds of Zhejiang Sci-Tech University (2020Y002); National Natural Science Foundation of China (81728012), China.

\section{Author contributions}

Study concept and design by W.-B.O., M.Z.L, and J.A.F; data acquisition by W.-B. O., M.Z.L., S.Z., N.B., A.K., L.X., T.C., A.M.E., and J.A.F.; analysis and interpretation of data by W.-B.O., M.Z.L, and J.A.F.; drafting of the manuscript by W.-B.O., M.Z.L, N.B., and J.A.F.; technical or material support by W.-B.O. and J.A.F.; study supervision by W.-B.O. and J.A.F.

Conflict of interest

The authors declare no competing interests.

\section{Publisher's note}

Springer Nature remains neutral with regard to jurisdictional claims in published maps and institutional affiliations.

Supplementary information The online version contains supplementary material available at https://doi.org/10.1038/s41389-021-00327-w.

Received: 12 September 2020 Revised: 26 March 2021 Accepted: 15 April 2021

Published online: 04 May 2021

\section{References}

1. Lee, C. H. et al. 14-3-3 fusion oncogenes in high-grade endometrial stromal sarcoma. Proc. Natl Acad. Sci. USA 109, 929-934 (2012).

2. Hoang, L., Chiang, S. \& Lee, C. H. Endometrial stromal sarcomas and related neoplasms: new developments and diagnostic considerations. Pathology 50, 162-177 (2018).

3. Conklin, C. M. \& Longacre, T. A. Endometrial stromal tumors: the new WHO classification. Adv. Anat. Pathol. 21, 383-393 (2014).

4. Mariño-Enriquez, A. et al. BCOR internal tandem duplication in high-grade uterine sarcomas. Am. J. Surg. Pathol. 42, 335-341 (2018). 
5. Kruse, A. J. et al. Aggressive behavior and poor prognosis of endometrial stromal sarcomas with YWHAE-FAM22 rearrangement indicate the clinical importance to recognize this subset. Int. J. Gynecol. Cancer 24, 1616-1622 (2014).

6. Gremel, G. et al. A prognosis based classification of undifferentiated uterine sarcomas: identification of mitotic index, hormone receptors and YWHAEFAM22 translocation status as predictors of survival. Int. J. Cancer 136, 1608-1618 (2015).

7. O'Meara, E. et al. Characterization of the chromosomal translocation $t(10 ; 17)$ (q22;p13) in clear cell sarcoma of kidney. J. Pathol. 27, 72-80 (2012).

8. Lee, C. H. et al. The clinicopathologic features of YWHAE-FAM22 endometrial stromal sarcomas: a histologically high-grade and clinically aggressive tumor. Am. J. Surg. Pathol. 36, 641-653 (2012).

9. Lee, C. H. et al. Cyclin D1 as a diagnostic immunomarker for endometrial stromal sarcoma with YWHAE-FAM22 rearrangement. Am. J. Surg. Pathol. 36, 1562-1570 (2012).

10. Gardino, A. K., Smerdon, S. J. \& Yaffe, M. B. Structural determinants of 14-3-3 binding specificities and regulation of subcellular localization of 14-3-3-ligand complexes: a comparison of the X-ray crystal structures of all human 14-3-3 isoforms. Semin. Cancer Biol. 16, 173-182 (2006).

11. Aitken, A. 14-3-3 proteins: a historic overview. Semin. Cancer Biol. 16, 162-172 (2006).

12. Mackintosh, C. Dynamic interactions between 14-3-3 proteins and phosphoproteins regulate diverse cellular processes. Biochem. J. 381, 329-342 (2004).

13. Zhang, W. The role of 14-3-3 proteins in gynecological tumors. Front. Biosci. 20, 934-945 (2015).

14. Kanai, F. et al. TAZ: a novel transcriptional co-activator regulated by interactions with 14-3-3 and PDZ domain proteins. EMBO J. 19, 6778-6791 (2000).

15. Zhao, B. et al. Inactivation of YAP oncoprotein by the Hippo pathway is involved in cell contact inhibition and tissue growth control. Gene. Dev. 21, 2747-2761 (2007).

16. Kofler, M. et al. Mediated nuclear import and export of TAZ and the underlying molecular requirements. Nat. Commun. 9, 4966 (2018).

17. Moreno-Vicente, R. et al. Caveolin-1 modulates mechanotransduction responses to substrate stiffness through actin-dependent control of YAP. Cell Rep. 25, 1622-1635.e1626 (2018).
18. Wang, S. et al. RN181 is a tumour suppressor in gastric cancer by regulation of the ERK/MAPK-cyclin D1/CDK4 pathway. J. Pathol. 248, 204-216 (2019).

19. Varga, A. et al. RAF1/BRAF dimerization integrates the signal from RAS to ERK and ROKalpha. Sci. signal. 10, eaai8482 (2017).

20. Nixon, B. R. et al. Nuclear localized Raf1 isoform alters DNA-dependent protein kinase activity and the DNA damage response. FASEB J. 33, 1138-1150 (2019).

21. Huang, $Y$. et al. Decoding the full picture of Raf1 function based on its interacting proteins. Oncotarget 8, 68329-68337 (2017).

22. Diedrich, B. et al. Discrete cytosolic macromolecular BRAF complexes exhibit distinct activities and composition. EMBO J. 36, 646-663 (2017).

23. Mielgo, A. et al. A MEK-independent role for CRAF in mitosis and tumor progression. Nat. Med. 17, 1641-1645 (2011).

24. Wang, S., Ghosh, R. N. \& Chellappan, S. P. Raf-1 physically interacts with Rb and regulates its function: a link between mitogenic signaling and cell cycle regulation. Mol. Cell. Biol. 18, 7487-7498 (1998).

25. Geil, W. M. \& Yen, A. Nuclear Raf-1 kinase regulates the CXCR5 promoter by associating with NFATC3 to drive retinoic acid-induced leukemic cell differentiation. FEBS J. 281, 1170-1180 (2014).

26. Ou, W. B. et al. Cyclin D1 is a mediator of gastrointestinal stromal tumor KITindependence. Oncogene 38, 6615-6629 (2019).

27. Deshpande, A., Sicinski, P. \& Hinds, P. W. Cyclins and cdks in development and cancer: a perspective. Oncogene 24, 2909-2915 (2005).

28. Jirawatnotai, S. et al. A function for cyclin D1 in DNA repair uncovered by protein interactome analyses in human cancers. Nature 474, 230-234 (2011).

29. Tobin, N. P. \& Bergh, J. Analysis of cyclin D1 in breast cancer: a call to arms Curr. Breast Cancer Rep. 4, 171-173 (2012).

30. de Boer, C. J. et al. Multiple breakpoints within the BCL-1 locus in B-cell lymphoma: rearrangements of the cyclin D1 gene. Cancer Res. 53, 4148-4152 (1993).

31. Verschoor, A. J., Warmerdam, F., Bosse, T., Bovee, J. \& Gelderblom, H. A remarkable response to pazopanib, despite recurrent liver toxicity, in a patient with a high grade endometrial stromal sarcoma, a case report. BMC Cancer 18, 92 (2018).

32. Rubin, B. P. et al. KIT activation is a ubiquitous feature of gastrointestinal stromal tumors. Cancer Res. 61, 8118-8121 (2001). 